\title{
Thymoquinone inhibits epithelial-mesenchymal transition in prostate cancer cells by negatively regulating the TGF- $\beta /$ Smad2/3 signaling pathway
}

\author{
BO KOU ${ }^{1 *}$, WEI LIU ${ }^{2 *}$, WEI ZHAO ${ }^{3 *}$, PENG DUAN $^{4}$, YANG YANG $^{1}$, QIUYUE YI $^{1}$, FENGWEI GUO ${ }^{1}$, \\ JIANPENG LI ${ }^{1}$, JINSONG ZHOU ${ }^{5,6}$ and QINGSHAN KOU ${ }^{7}$
}

\begin{abstract}
Departments of ${ }^{1}$ Cadiovascular Surgery, ${ }^{2}$ Urology, ${ }^{3}$ General Surgery and ${ }^{4}$ The First Affiliated Hospital of the Medical School, Xi'an Jiaotong University, Xi'an, Shaanxi 710061; ${ }^{5}$ Department of Human Anatomy, Histology and Embryology,

School of Basic Medical Sciences, Xi'an Jiaotong University Health Science Center, Xi'an, Shaanxi 710061;

${ }^{6}$ Key Laboratory of Environment and Genes Related to Diseases, Xi'an Jiaotong University, Ministry of Education of China, Xi'an, Shaanxi 710061; ${ }^{7}$ Medical Center, First People's Hospital of Xianyang, Xianyang, Shaanxi 712000, P.R. China
\end{abstract}

Received April 11, 2017; Accepted September 20, 2017

DOI: 10.3892/or.2017.6012

\begin{abstract}
Thymoquinone, a major ingredient of black seed oil (Nigella sativa), has been shown to exhibit anticancer capacity in various types of cancers. However, there are few studies concerning the correlation between thymoquinone and epithelial-to-mesenchymal transition (EMT) in prostate cancer. In the present study, we firstly found that thymoquinone showed antimetastatic capacity in prostate cancer DU145 and PC3 cells. Additionally, thymoquinone reversed EMT by increasing E-cadherin expression and decreasing vimentin and Slug expression in a concentration-dependent manner. Recent studies have shown that the transforming growth factor- $\beta$ (TGF- $\beta$ ) signaling pathway may be associated with EMT. Intriguingly, the expression of TGF- $\beta, \operatorname{Smad} 2$ and $\operatorname{Smad} 3$ at the mRNA and protein levels was notably reduced upon thymoquinone treatment in prostate cancer DU145 and PC3 cells. Subsequently, we confirmed that thymoquinone repressed metastasis and EMT of prostate cancer through downregulation of the TGF- $\beta / \mathrm{Smad} 2 / 3$ signaling pathway, which may be partially reversed by TGF- $\beta$ overexpression. In summary, our findings demonstrated that thymoquinone suppressed the metastatic phenotype and reversed EMT of prostate cancer
\end{abstract}

Correspondence to: Professor Jinsong Zhou, Department of Human Anatomy, Histology and Embryology, School of Basic Medical Sciences, Xi'an Jiaotong University Health Science Center, 76 West Yanta Road, Xi'an, Shaanxi 710061, P.R. China

E-mail: zjs301@mail.xjtu.edu.cn

Dr Qingshan Kou, Medical Center, First People's Hospital of Xianyang, Xianyang, Shaanxi 712000, P.R. China

E-mail: 492526094@qq.com

*Contributed equally

Key words: thymoquinone, prostate cancer, migration, invasion, epithelial-mesenchymal transition, TGF- $\beta$ cells by negatively regulating the TGF- $\beta / \mathrm{Smad} 2 / 3$ signaling pathway. These findings suggest that thymoquinone is a potential therapeutic agent against prostate cancer which functions by targeting TGF- $\beta$.

\section{Introduction}

Prostate cancer is the most lethal urogenital system malignancy in men, accounting for an estimated 161,360 new cases and 26,730 deaths in the US in 2017 (1). Although great improvement has been made in the efficacy of therapeutic methods, most prostate cancer develops into a metastatic pattern, which is a major obstacle for the treatment of prostate cancer (2). Recent research has reported that epithelial-to-mesenchymal transition (EMT) is a necessity for the invasion-metastasis cascade of cancer (3). EMT is a complicated process which involves the loss of epithelial characteristics and acquisition of a mesenchymal phenotype, contributing to the initiation of the metastatic cascade (4). Given that there are few effective therapeutic methods for the treatment of cancer metastasis, the development of novel therapeutic agents against prostate cancer is critically needed.

Thymoquinone (TQ), a major ingredient of black seed oil (Nigella sativa), has been shown to exhibit broad pharmacologic effects, such as anti-inflammatory (5) and antioxidant activity (6). Moreover, thymoquinone has been reported to possess antineoplastic activity against various cancers, including pancreatic (7), lung (8) and colon cancer (9) and leukemia (10). Studies have shown that thymoquinone inhibits the growth and induces cell apoptosis and cycle arrest, as well as represses metastasis and angiogenesis, which involves Akt (11), NF- $\kappa \mathrm{B}$ signaling (12), mitogen-activated protein kinase (MAPK) (13) and STAT3 signaling pathways (14).

In prostate cancer $\mathrm{C} 4-2 \mathrm{~B}$ and $\mathrm{PC} 3$ cells, thymoquinone was found to suppress proliferation via the accumulation of reactive oxygen species (ROS) and reduction in the glutathione (GST) level (15). However, there is no study concerning the correlation between thymoquinone and EMT in prostate 
cancer. In addition, the underlying mechanism by which thymoquinone suppresses the metastatic phenotype has not yet been elucidated. The present study aimed to explore the potential capacity of thymoquinone to reverse EMT in prostate cancer cells and the related mechanism.

\section{Materials and methods}

Reagents and cell culture. Thymoquinone $\left(\mathrm{C}_{10} \mathrm{H}_{12} \mathrm{O}_{2}\right)$ was purchased from Sigma-Aldrich (St. Louis, MO, USA) and dissolved in dimethyl sulfoxide (DMSO). Stock solutions were stored at $-20^{\circ} \mathrm{C}$. Antibodies against transforming growth factor- $\beta$ (TGF- $\beta$ ), Smad2, Smad3, E-cadherin, vimentin, Slug and $\beta$-actin were purchased from Cell Signaling Technology, Inc. (Beverly, MA, USA). 3-(4,5-Dimethylthiazol-2-yl)-2,5diphenyltetrazolium bromide (MTT) was purchased from Sigma Chemical Co. (St. Louis, MO, USA).

Human prostate cancer cell lines DU145 and PC3 were purchased from the American Type Culture Collection (ATCC; Manassas, VA, USA). These two cell lines were cultured in RPMI-1640 medium supplemented with $10 \%$ fetal bovine serum (Gibco, Grand Island, NY, USA), $100 \mu \mathrm{g} / \mathrm{ml}$ streptomycin and $100 \mathrm{U} / \mathrm{ml}$ penicillin (Invitrogen, Carlsbad, $\mathrm{CA}, \mathrm{USA})$ at $37^{\circ} \mathrm{C}$ in a humidified incubator with $5 \% \mathrm{CO}_{2}$.

Cell proliferation assay. A modified MTT assay was used to evaluate cell proliferation. In brief, DU145 and PC3 cells were seeded at a density of $1.2 \times 10^{4} /$ well in 96 -well plates with $90 \%$ density and subsequently exposed to increasing concentrations of thymoquinone for $24 \mathrm{~h}$. Then, $20 \mu \mathrm{l}$ MTT dye solution $(5.0 \mathrm{mg} / \mathrm{ml})$ was mixed with $180 \mu \mathrm{l}$ medium and added to each well. After incubation at $37^{\circ} \mathrm{C}$ for $4 \mathrm{~h}$, the culture medium was wiped out and the cells were lysed with DMSO to dissolve the formazan crystals. The optical density (OD) of each well was evaluated at $490 \mathrm{~nm}$ wavelength using a 96-well microplate reader (Bio-Rad, Hercules, CA, USA). The growth inhibitory rate was calculated as: [(OD $490_{\text {control cells }}$ - OD $\left.490_{\text {treated cells }}\right) /$ OD $\left.490_{\text {control cells }}\right]$ x 100 . The experiments were performed in triplicate.

Wound healing assay. Wound healing assay was performed to detect the effect of thymoquinone on cell migration. Prostate cancer DU145 or PC3 cells were seeded onto 6-well plates. When the cell density reached above $90 \%$, scratch wounds were made across the monolayer using the tip of a $200-\mu 1$ pipette. Then, the wounded cultures were incubated in a serumfree medium with thymoquinone treatment at different times $(0,24 \mathrm{~h})$, and five random fields (magnification, x100) were subsequently chosen from each scratch wound and observed by microscopy to evaluate the migratory capacity. The experiments were performed in triplicate.

Transwell migration assay. Transwell migration assay is another method to evaluate cell migratory ability. It was performed using prostate cancer DU145 and PC3 cells after thymoquinone treatment. Cells $\left(4 \times 10^{4}\right)$ with $200 \mu 1$ serum-free medium were seeded onto the upper chamber, while $800 \mu \mathrm{l}$ of medium supplemented with $10 \%$ fetal calf serum was added to the lower chamber. After certain incubation at $37^{\circ} \mathrm{C}$, the adherent cells on the top chambers were wiped off with a cotton swab. The migrated cells on the lower surface of the filter were then fixed with $4 \%$ paraformaldehyde and stained with $0.1 \%$ crystal violet (Beyotime, Shanghai, China). Cells were then counted in five randomly chosen visual fields using a microscope at a magnification of $\mathrm{x} 100$. The experiments were performed in triplicate.

Matrigel invasion assay. The impact of thymoquinone on the invasion of prostate cancer cells was evaluated by Matrigel invasion assay using a Millicell chamber (Millipore, Billerica, MA, USA). The membrane (polycarbonic membrane, $8 \mu \mathrm{m}$ pore size) in the top chamber was pretreated with $50 \mu 1$ Matrigel (Matrigel, serum-free medium 1:5). After incubation at $37^{\circ} \mathrm{C}$ for $5 \mathrm{~h}$, the cells $\left(10 \times 10^{4}\right)$ in $200 \mu \mathrm{l}$ serum-free medium were treated with thymoquinone according to the instructions of the Transwell migration assay.

Quantitative real-time PCR assay. DU145 and PC3 cells were treated with different concentrations of thymoquinone and the total RNA was extracted using TRIzol reagent (Invitrogen, Carlsbad, CA, USA) according to the manufacturer's protocol. Complementary DNA (cDNA) was subsequently synthesized using a PrimerScript RT reagent kit (Takara, Dalian, China). Then, the relative levels of target gene messenger RNA (mRNA) transcript were measured by quantitative real-time PCR assay (qRT-PCR) using the SYBR-Green Master Mix. The sequences of primers for the PCR amplification were forward, 5'-GGCCAGATCCTGTCCAAGC-3' and reverse, 5'-GTGGGTTTCCACCATTAGCAC-3' for TGF- $\beta$ (201 bp); 5'-CGTCCATCTTGCCATTCACG-3' and reverse, 5'-CTC AAGCTCATCTAATCGTCCTG-3' for $\operatorname{Smad} 2$ (182 bp); 5'-TGGACGCAGGTTCTCCAAAC-3' and reverse, 5'-CCG GCTCGCAGTAGGTAAC-3' for Smad3 (90 bp); forward 5'-CGAGAGCTACACGTTCACGG-3' and reverse, 5'-GGG TGTCGAGGGAAAAATAGG-3' for E-cadherin (119 bp); forward, 5'-GACGCCATCAACACCGAGTT-3' and reverse, 5'-CTTTGTCGTTGGTTAGCTGGT-3' for vimentin (238 bp); forward, 5'-CGAACTGGACACACATACAGTG-3' and reverse, 5'-CTGAGGATCTCTGGTTGTGGT-3' for Slug (87 bp); forward, 5'-CATGTACGTTGCTATCCAGGC-3' and reverse, 5'-CTCCTTAATGTCACGCACGAT-3' for $\beta$-actin (250 bp). All the experiments were performed in triplicate and calculated on the basis of the $\Delta \Delta \mathrm{Ct}$ method. The $\mathrm{n}$-fold change in mRNA expression was evaluated according to the method of $2^{-\Delta \Delta C t}$.

Western blotting. Briefly, prostate cancer DU145 and PC3 cells were collected after thymoquinone treatment, and lysed on ice for $10 \mathrm{~min}$ with a lysis buffer $[10 \mathrm{mmol} / 1 \mathrm{Tris}-\mathrm{HCl}$ (pH 7.4), $150 \mathrm{mmol} / 1 \mathrm{NaCl}, 0.1 \%$ sodium dodecyl sulfate (SDS), $1 \mathrm{mmol} / 1$ ethylenediaminetetraacetic acid, $1 \mathrm{mmol} / \mathrm{l}$ ethylene glycol tetraacetic acid, $0.3 \mathrm{mmol} / 1$ phenylmethylsulfonyl fluoride, $0.2 \mathrm{mmol} / 1$ sodium orthovanadate, $1 \% \mathrm{NP}-40$, $10 \mathrm{mg} / \mathrm{ml}$ leupeptin and $10 \mathrm{mg} / \mathrm{ml}$ aprotinin]. After centrifugation and denaturation, the clarified protein lysates ( 30-60 $\mu \mathrm{g})$ were subjected to SDS-polyacrylamide gel electrophoresis (10 or $15 \%$ ) and transferred to polyvinylidene membranes (Millipore). Membranes were subsequently probed with antibodies against TGF- $\beta$, Smad2, Smad3, E-cadherin, vimentin, Slug and $\beta$-actin overnight at $4^{\circ} \mathrm{C}$. The immunoreactive bands 

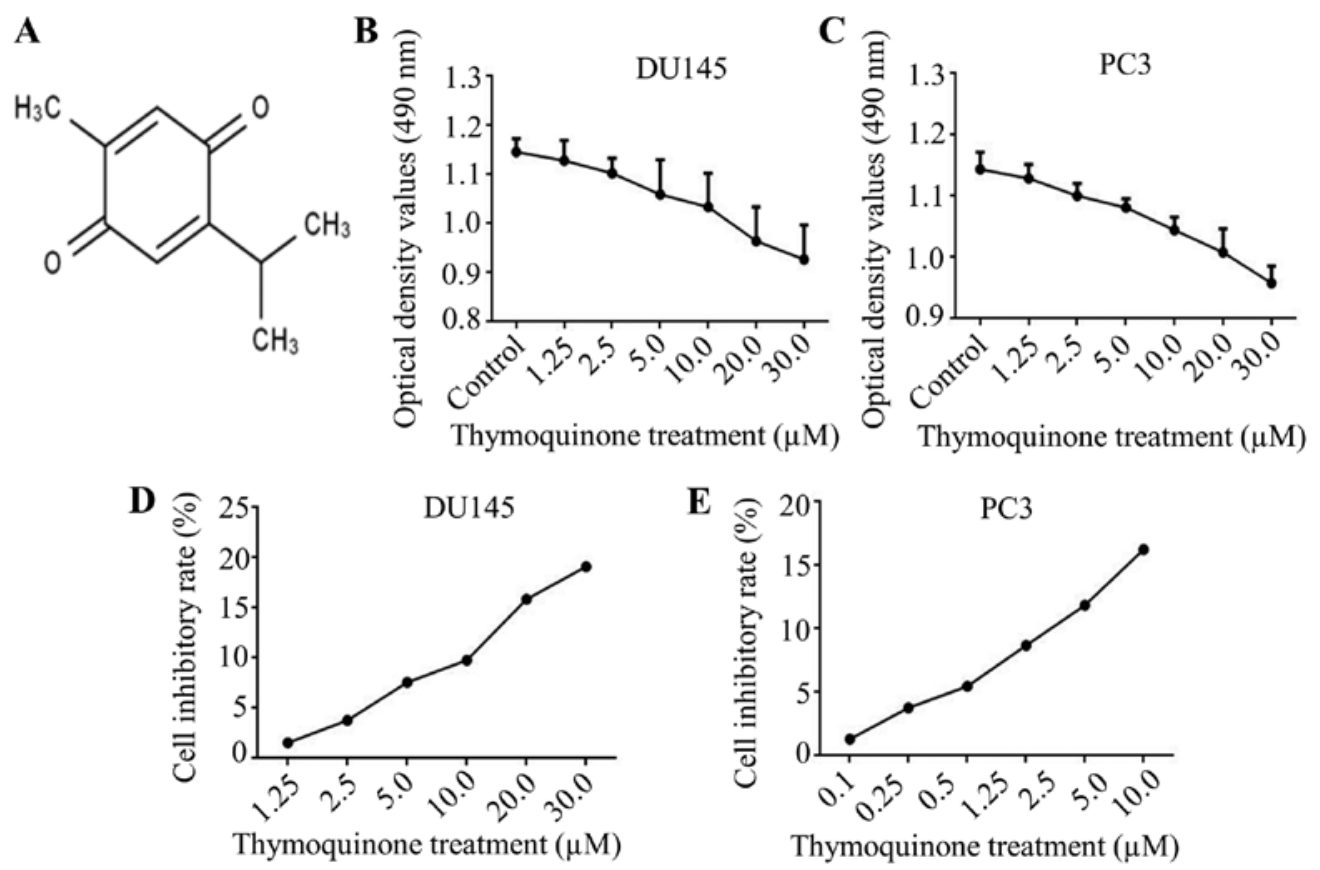

Figure 1. Thymoquinone inhibits the proliferation of prostate cancer cells. (A) Chemical structure of thymoquinone. (B-E) After DU145 and PC3 cells at $90 \%$ density were treated with various concentrations of thymoquinone for $24 \mathrm{~h}$, the viability of the prostate cancer cells was evaluated by MTT assays. The optical density and cell inhibitory rate of three independent experiments is shown for (B and D) DU145 and (C and E) PC3 cells.

were then washed and incubated with horseradish peroxidase (HRP)-conjugated secondary antibody at room temperature $\left(25^{\circ} \mathrm{C}\right)$. Ultimately, the protein bands were visualized with ECL substrate and exposed to X-ray film.

Plasmid transfection. TGF- $\beta$ cDNA was cloned into the pcDNA3.1 vector. When prostate cancer DU145 or PC3 cells reached $70-80 \%$ confluency for plasmid transfection, the cells were then transfected with X-tremeGENE HP DNA transfection reagent (Roche, Mannheim, Germany) for $48 \mathrm{~h}$ following the manufacturer's instructions, and prepared for the subsequent turnover experiments.

Statistical analysis. All statistical analyses were evaluated by GraphPad Prism (version 5.0) software (GraphPad Software, Inc., La Jolla, CA, USA), and Student's t-test (two-sided) was used for comparisons involving only two groups. A value of $\mathrm{P}<0.05$ was identified as a statistical significant difference.

\section{Results}

Thymoquinone inhibits the proliferation of prostate cancer cells. Firstly, the chemical structure of thymoquinone is presented in Fig. 1A. In view of the metastatic potential, DU145 and PC3 cells were chosen for the next research. Subsequently, to exclude the effect of thymoquinone on growth inhibition in prostate cancer cells, a modified MTT assay was used. The results demonstrated that the growth of prostate cancer DU145 and PC3 cells at a density $>90 \%$ was notably repressed upon thymoquinone treatment at concentrations $\geq 10.0 \mu \mathrm{M}$ (Fig. 1B-E). Thymoquinone at $10 \mu \mathrm{M}$ was used as the adequate concentration in the subsequent research, to exclude interference from growth inhibition by thymoquinone.
Thymoquinone (TQ) inhibits the migratory and invasive capacity of prostate cancer DU145 and PC3 cells. To explore the effect of thymoquinone on migratory and invasive abilities in prostate cancer cells, a wound healing assay was used to verify the antimetastatic effect of thymoquinone. The results indicated that the scratch width was much wider upon thymoquinone treatment than that in the control group in the DU145 and PC3 cells, suggesting the effective antimetastatic activity of thymoquinone on prostate cancer cells (Fig. 2A and B). To further confirm the effects of thymoquinone on the metastatic phenotype of prostate cancer cells, Transwell migration and Matrigel invasion assays were carried out. We found that the migratory ability of DU145 cells was significantly decreased by thymoquinone treatment $(\mathrm{P}<0.05)$ (Fig. 2C). Similar results were noted in the prostate cancer PC3 cells treated with thymoquinone (Fig. 2D). Next, we explored the effect of thymoquinone on the invasiveness of prostate cancer cells and our findings indicated that thymoquinone significantly suppressed the invasive capability of the DU145 and PC3 cells ( $\mathrm{P}<0.05)$ (Fig. 2C and D).

Taken together, the results from the wound healing and Transwell assays revealed that thymoquinone had a strong antimetastatic effect on human prostate cancer cells.

Thymoquinone represses the expression of EMT markers in prostate cancer cells. EMT has been widely reported to be associated with the ability of migration and invasion in various cancers. In view of that, we first examined the expression levels of EMT markers including E-cadherin, vimentin and Slug by quantitative real-time PCR after treatment of DU145 and PC3 cells with 2.5, 5.0 and $10 \mu \mathrm{M}$ thymoquinone for $24 \mathrm{~h}$. We found that the mRNA level of E-cadherin was notably upregulated upon thymoquinone treatment in a concentration-dependent manner, whereas the expression levels of vimentin and Slug at mRNA levels were significantly downregulated $(\mathrm{P}<0.05)$ 
A
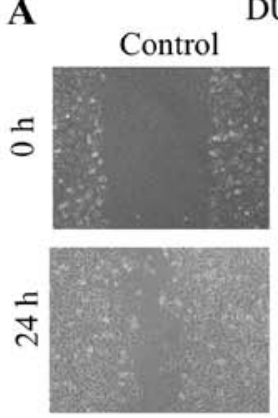

DU145

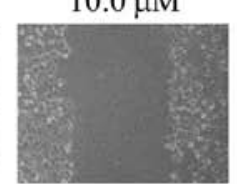

B
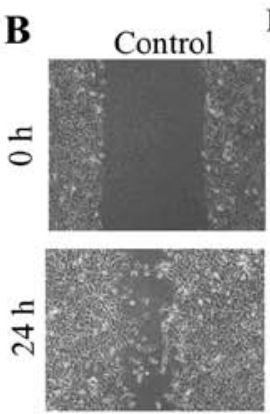

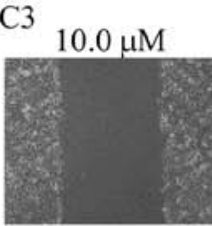

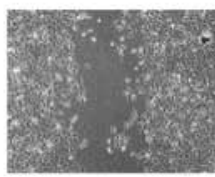

C

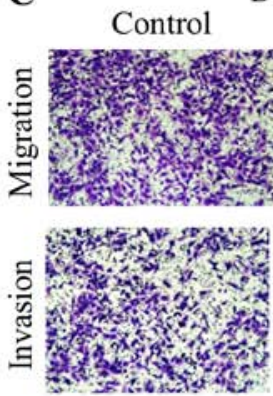

DU145

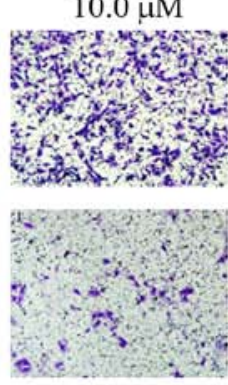

PC3
$10.0 \mu \mathrm{M}$
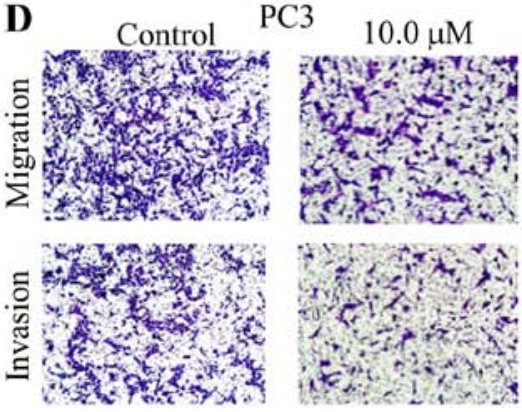

DU145

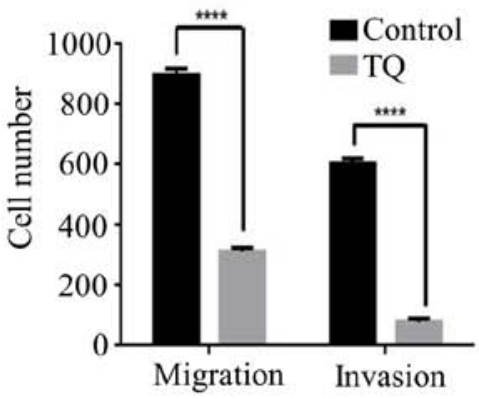

$\mathrm{PC} 3$

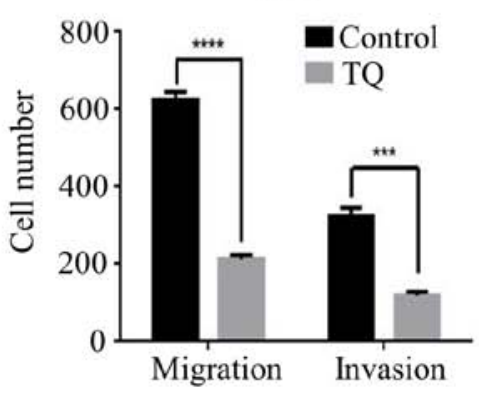

Figure 2. Thymoquinone (TQ) inhibits the migratory and invasive capacity of prostate cancer DU145 and PC3 cells. The width of scratches was detected with or without thymoquinone treatment at 0 and $24 \mathrm{~h}$ in prostate cancer (A) DU145 and (B) PC3 cells. (C and D) In addition, using Transwell migration and Matrigel invasion assays, the numbers of migrated and invaded (C) DU145 and (D) PC3 cells treated with $10 \mu \mathrm{M}$ thymoquinone/chamber were determined and compared with these numbers in the control group from three independent experiments $\left({ }^{* * *} \mathrm{P}<0.001\right.$ and $\left.{ }^{* * * *} \mathrm{P}<0.0001\right)$.

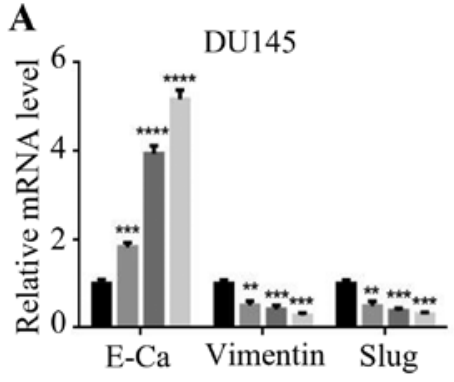

C
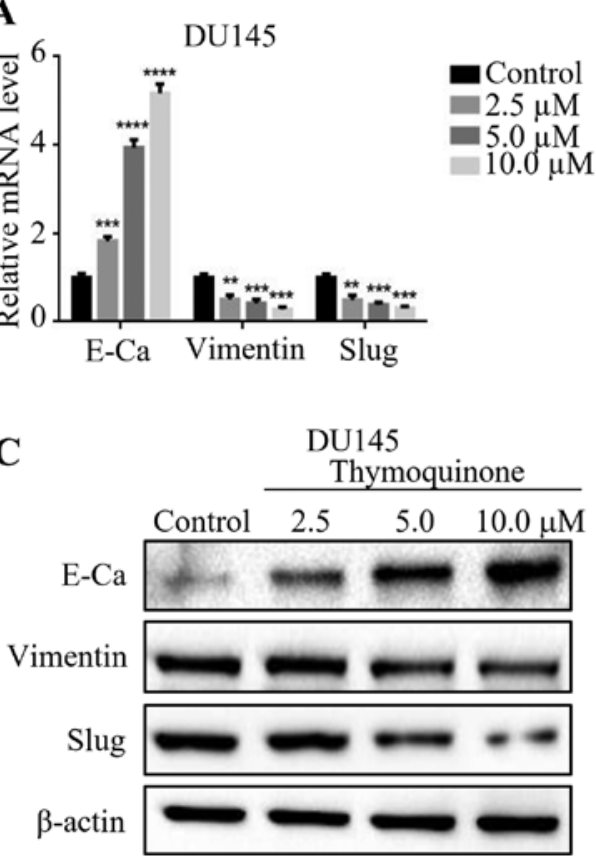

B

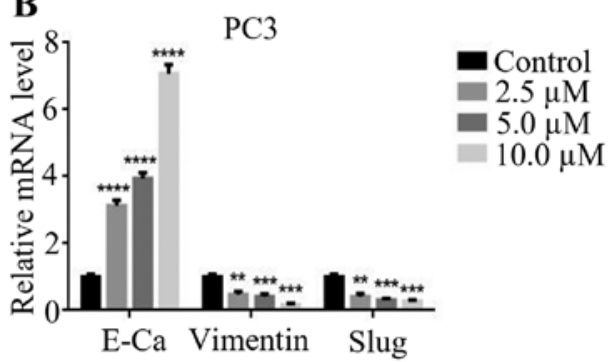

D

PC3

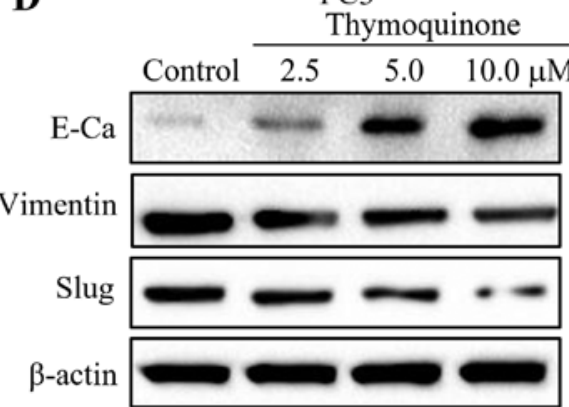

Figure 3. Thymoquinone suppresses the expression of epithelial-mesenchymal transition markers in prostate cancer cells. Quantitive real-time PCR was used to examine the expression of E-cadherin, vimentin, Slug and $\beta$-actin in prostate cancer (A) DU145 and (B) $\mathrm{PC} 3$ cell lines. ${ }^{* * *} \mathrm{P}<0.01,{ }^{* * * *} \mathrm{P}<0.001$ and ${ }^{* * * * *} \mathrm{P}<0.0001$. (C) DU145 and (D) PC3 cells treated with thymoquinone were subjected to western blotting for E-cadherin (E-Ca), vimentin, Slug and $\beta$-actin. Representative protein bands from three experiments are shown.

(Fig. 3A and B). Consistent with the above results, western blot analysis showed that thymoquinone decreased the expression levels of the mesenchymal markers including vimentin and Slug, while treatment with thymoquinone increased the expression of E-cadherin (Fig. 3C and D) in the DU145 and
PC3 cells. The results revealed that thymoquinone reversed EMT in prostate cancer cells.

Thymoquinone reduces the expression of TGF- $\beta$, Smad 2 and Smad3 in prostate cancer cells. Studies have shown that the 
A

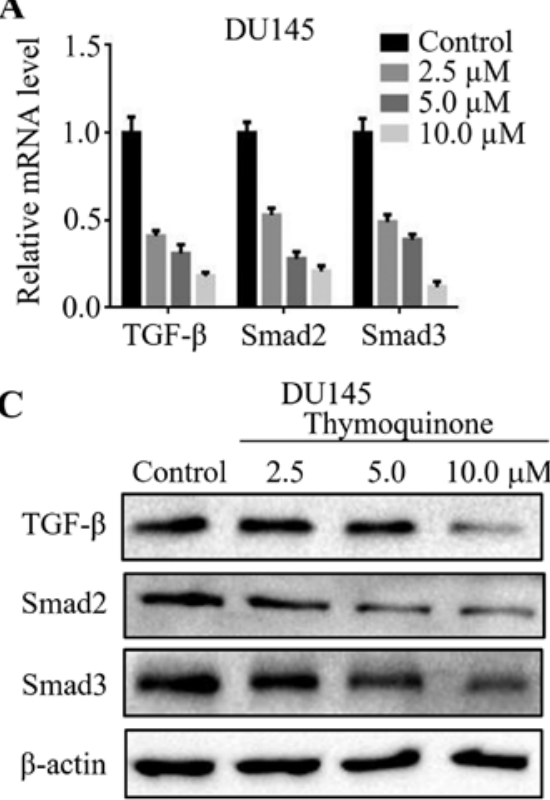

B

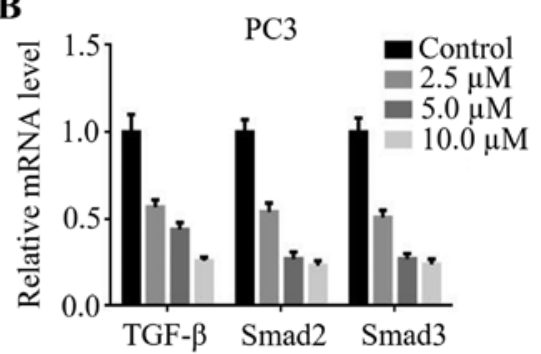

D

PC3

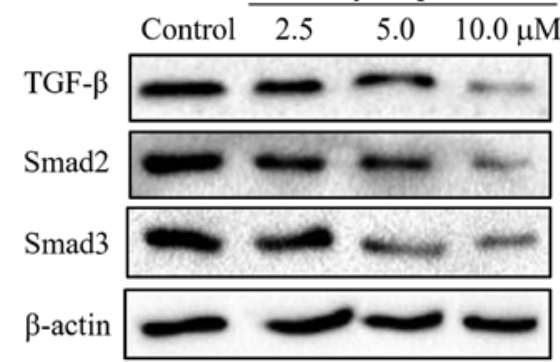

Figure 4. Thymoquinone reduces the expression of TGF- $\beta$, Smad2 and Smad3 in prostate cancer cells. (A and B) Quantitative real-time PCR was used to examine the expression of TGF- $\beta$, Smad2 and Smad3 in prostate cancer (A) DU145 and (B) PC3 cells. Furthermore, TGF- $\beta$, Smad 2 and Smad3 levels in prostate cancer (C) DU145 and (D) PC3 cells treated with thymoquinone were determined by western blotting.

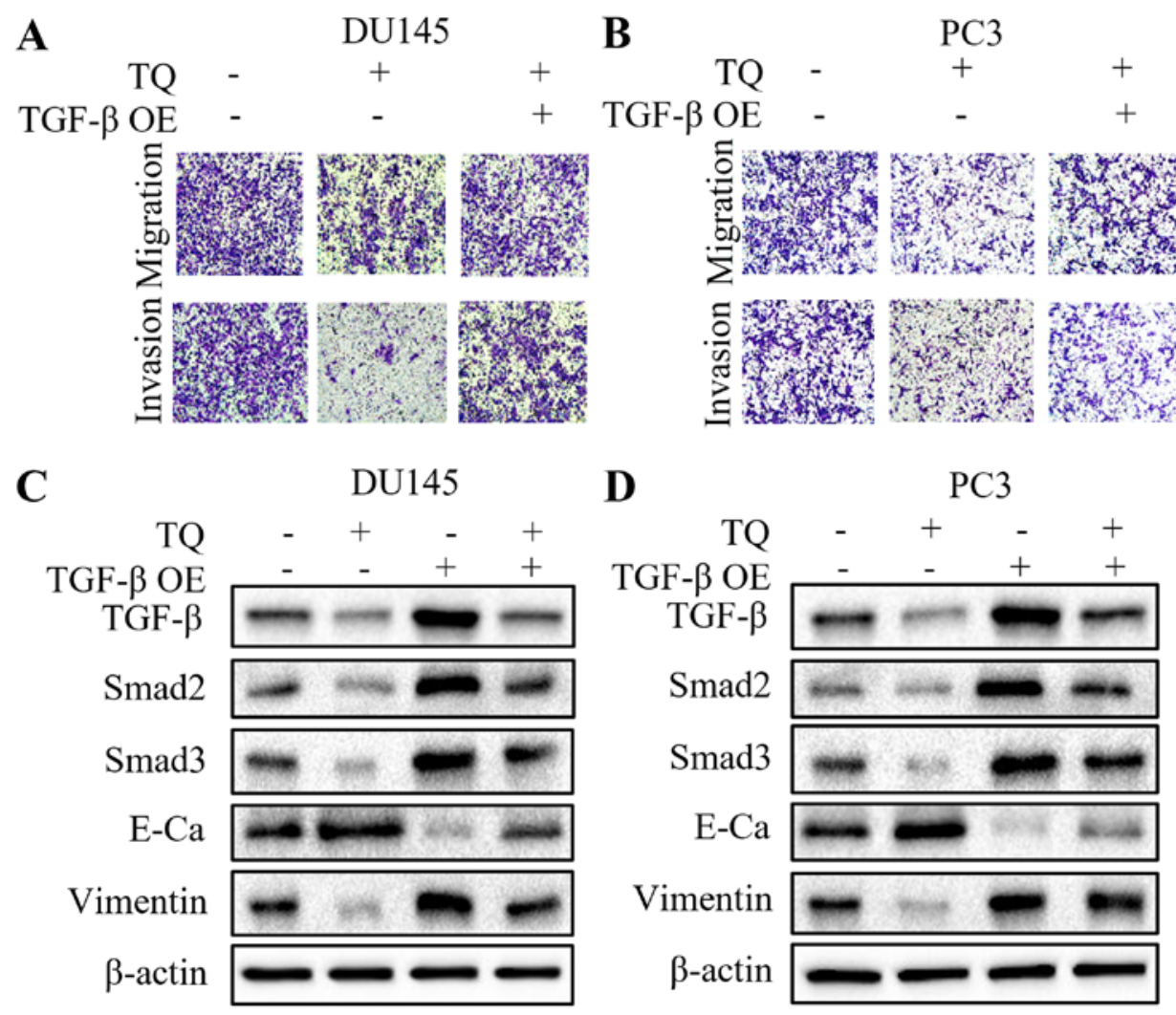

Figure 5. Overexpression (OE) of TGF- $\beta$ attenuates the antitumor capacity of thymoquinone (TQ) in prostate cancer cells. Transwell migration and Matrigel invasion assays were performed on (A) DU145 and (B) PC3 cells following combination treatment of TQ and TGF- $\beta$ overexpression. Five random fields were chosen and visualized by microscopy to assess the cell migratory and invasive capacity. Western blotting was performed to evaluate the levels of TGF- $\beta$, Smad2, Smad3, E-cadherin (E-Ca), vimentin and $\beta$-actin protein upon various treatments in the (C) DU145 and (D) PC3 cells. Representative protein bands of three experiments are shown.

TGF- $\beta$ signaling pathway is implicated in cancer invasion, metastasis and angiogenesis (16). To validate the underlying mechanism involved in the antimetastatic effect of thymo- quinone in prostate cancer cells, we detected the expression levels of TGF- $\beta$, Smad 2 and Smad 3 in the prostate cancer cells upon thymoquinone treatment. Notably, thymoquinone down- 
regulated TGF- $\beta, \operatorname{Smad} 2$ and $\mathrm{Smad} 3$ expression at the mRNA level in a concentration-dependent manner (Fig. 4A and B). In accordance with the above results, the protein levels of TGF- $\beta$, Smad 2 and Smad3 in the DU145 and PC3 cells were significantly reduced following thymoquinone treatment $(\mathrm{P}<0.05)$ (Fig. 4C and D).

Overexpression of TGF- $\beta$ attenuates the antitumor capacity of thymoquinone in prostate cancer cells. To further explore whether the TGF- $\beta$ signaling pathway is involved in the antimetastatic effect of thymoquinone on prostate cancer cells, the TGF- $\beta$ plasmid was transiently transfected into DU145 and PC3 cells to overexpress TGF- $\beta$. Intriguingly, overexpression of TGF- $\beta$ partially restored the migratory and invasive capacities of the prostate cancer cells, which was inhibited by thymoquinone (Fig. 5A and B). These findings suggested that TGF- $\beta$ may play a crucial role in the antimetastatic activity of thymoquinone in prostate cancer. Subsequently, we found a marked reversal of the elevated E-cadherin level, and reduced vimentin, Smad2 and Smad3 levels following the combined treatment of thymoquinone and TGF- $\beta$ overexpression, compared with thymoquinone treatment alone (Fig. 5C and D). These results revealed that the antimetastatic capacity of thymoquinone in prostate cancer may be mediated by the TGF- $\beta$ signaling pathway.

\section{Discussion}

Numerous studies have shown that thymoquinone is able to impede tumor progression in a variety of tumors. It is reported that in prostate cancer PC3 cells, thymoquinone may suppress tumor angiogenesis and growth by downregulating AKT and extracellular signal-regulated kinase (ERK) signaling (17). Additionally, thymoquinone notably suppressed DNA synthesis and proliferation in prostate cancer LNCaP, C4-2B, DU145 and PC3 cell lines via inactivation of the androgen receptor $(A R)$ and $E 2 F 1$, a regulator of cell viability (18). In addition, it has been found that thymoquinone could potentiate the cytotoxic and pro-apoptotic effect of zoledronic acid (ZA) in prostate cancer PC3 and DU145 cells (19). In the present study, we indeed confirmed the cytotoxic effect of thymoquinone on prostate cancer DU145 and PC 3 cells. The subsequent wound healing and Transwell assays revealed that thymoquinone notably suppressed the metastatic phenotype of prostate cancer cells. Then, we detected the mRNA and protein levels of E-cadherin, vimentin and Slug, hallmarks of epithelial-tomesenchymal transition (EMT). As expected, thymoquinone significantly increased the expression of E-cadherin, while decreased the expression of vimentin and Slug at the mRNA and protein levels using quantitative real-time PCR and western blotting, suggesting that thymoquinone reversed EMT in prostate cancer DU145 and PC3 cells. Meanwhile, other studies have demonstrated that thymoquinone decreased the expression of Twistl at the transcriptional level, leading to the reversal of EMT in MDA-MB-435, BT549 and HeLa cells. In addition, thymoquinone downregulated $\mathrm{N}$-cadherin, Snail and Slug expressions in bladder cancer T24 cells, which were partially in accordance with our results (20).

The TGF- $\beta$ signaling pathway has been confirmed to regulate malignancy progression and metastasis (21). It is reported that TGF- $\beta$ may participate in the regulation of collagen (22), fibronectin (23), laminin (24) and MMP-9 (25), to affect migratory and invasive capacity. Accumulating evidence shows that Chinese herbal medicines, such as curcumin and anthocyanidins, reverse EMT and suppress metastasis via the downregulation of the TGF- $\beta / \mathrm{Smad} 2 / 3$ signaling pathway $(26,27)$. Our findings revealed a marked dose-dependent reduction in TGF- $\beta$, Smad 2 and Smad 3 levels upon thymoquinone treatment in prostate cancer DU145 and PC3 cells, which was confirmed by quantitative real-time PCR and western blotting. To further validate that the TGF- $\beta /$ Smad $2 / 3$ signaling pathway participates in the reversal of EMT by thymoquinone in prostate cancer, a TGF- $\beta$ overexpression plasmid was transiently transfected into DU145 and PC3 cells. The results demonstrated that overexpression of TGF- $\beta$ impaired the antimetastatic effect of thymoquinone and partially abrogated the induction of mesenchymal-toepithelial transition (MET) by thymoquinone, as evidenced by the reversal of the elevated E-cadherin level and decreased vimentin, Smad2 and Smad3 levels. These results indicated that the inhibitory effect of thymoquinone on EMT may be mediated by the TGF- $\beta / \operatorname{Smad} 2 / 3$ signaling pathway.

In summary, our study confirmed that thymoquinone suppressed the metastatic phenotype and inhibited EMT in prostate cancer cells by negatively regulating the TGF- $\beta$ / Smad2/3 signaling pathway. In addition, these findings suggest that thymoquinone is a potential therapeutic agent against prostate cancer which functions by targeting TGF- $\beta$

\section{Acknowledgements}

The present study was partially supported by the National Natural Science Foundation of China (no. 81602562), the International Science and Technology Cooperative Project of Shaanxi Province (no. 2017KW-063), the Fundamental Research Funds for the Central University of Xi'an Jiaotong University (no. 1191329722), and the Institutional Scientific Development Foundation of the First Affiliated Hospital of Xi'an Jiaotong University (no. 2015YK17).

\section{References}

1. Siegel RL, Miller KD and Jemal A: Cancer Statistics, 2017. CA Cancer J Clin 67: 7-30, 2017.

2. Leyh-Bannurah SR, Gazdovich S, Budäus L, Zaffuto E, Briganti A, Abdollah F, Montorsi F, Schiffmann J, Menon M, Shariat SF, et al: Local therapy improves survival in metastatic prostate cancer. Eur Urol 72: 118-124, 2017.

3. Mao XY, Li QQ, Gao YF, Zhou HH, Liu ZQ and Jin WL: Gap junction as an intercellular glue: Emerging roles in cancer EMT and metastasis. Cancer Lett 381: 133-137, 2016.

4. Cha YH, Yook JI, Kim HS and Kim NH: Catabolic metabolism during cancer EMT. Arch Pharm Res 38: 313-320, 2015.

5. Hossen MJ, Yang WS, Kim D, Aravinthan A, Kim JH and Cho JY: Thymoquinone: An IRAK1 inhibitor with in vivo and in vitro anti-inflammatory activities. Sci Rep 7: 42995, 2017.

6. Dur A, Kose H, Kocyigit A, Kocaman O, Ismayilova M and Sonmez FC: The anti-inflammatory and antioxidant effects of thymoquinone on ceruleine induced acute pancreatitis in rats. Bratisl Lek Listy 117: 614-618, 2016.

7. Relles D, Chipitsyna GI, Gong Q, Yeo CJ and Arafat HA: Thymoquinone promotes pancreatic cancer cell death and reduction of tumor size through combined inhibition of histone deacetylation and induction of histone acetylation. Adv Prev Med 2016: 1407840, 2016. 
8. Acharya BR, Chatterjee A, Ganguli A, Bhattacharya S and Chakrabarti G: Thymoquinone inhibits microtubule polymerization by tubulin binding and causes mitotic arrest following apoptosis in A549 cells. Biochimie 97: 78-91, 2014.

9. Mohamed AM, Refaat BA, El-Shemi AG, Kensara OA, Ahmad J and Idris S: Thymoquinone potentiates chemoprotective effect of Vitamin D3 against colon cancer: A pre-clinical finding. Am J Transl Res 9: 774-790, 2017.

10. Salim LZ, Othman R, Abdulla MA, Al-Jashamy K, Ali HM, Hassandarvish P, Dehghan F, Ibrahim MY, Omer FA and Mohan S: Thymoquinone inhibits murine leukemia WEHI-3 cells in vivo and in vitro. PLoS One 9: e115340, 2014

11. Xu D, Ma Y, Zhao B, Li S, Zhang Y, Pan S, Wu Y, Wang J, Wang D, Pan $\mathrm{H}$, et al: Thymoquinone induces $\mathrm{G} 2 / \mathrm{M}$ arrest, inactivates PI3K/Akt and nuclear factor- $\kappa \mathrm{B}$ pathways in human cholangiocarcinomas both in vitro and in vivo. Oncol Rep 31: 2063-2070, 2014.

12. Zhang L, Bai Y and Yang Y: Thymoquinone chemosensitizes colon cancer cells through inhibition of NF- $\mathrm{KB}$. Oncol Lett 12 2840-2845, 2016.

13. Woo CC, Hsu A, Kumar AP, Sethi G and Tan KH: Thymoquinone inhibits tumor growth and induces apoptosis in a breast cancer xenograft mouse model: The role of p38 MAPK and ROS. PLoS One 8: e75356, 2013.

14. Zhu WQ, Wang J, Guo XF, Liu Z and Dong WG: Thymoquinone inhibits proliferation in gastric cancer via the STAT3 pathway in vivo and in vitro. World J Gastroenterol 22: 4149-4159, 2016.

15. Koka PS, Mondal D, Schultz M, Abdel-Mageed AB and Agrawal KC: Studies on molecular mechanisms of growth inhibitory effects of thymoquinone against prostate cancer cells: Role of reactive oxygen species. Exp Biol Med 235: 751-760, 2010.

16. Chen W, Zhou S, Mao L, Zhang H, Sun D, Zhang J, Li J and Tang JH: Crosstalk between TGF- $\beta$ signaling and miRNAs in breast cancer metastasis. Tumour Biol 37: 10011-10019, 2016

17. Yi T, Cho SG, Yi Z, Pang X, Rodriguez M, Wang Y, Sethi G, Aggarwal BB and Liu M: Thymoquinone inhibits tumor angiogenesis and tumor growth through suppressing AKT and extracellular signal-regulated kinase signaling pathways. Mol Cancer Ther 7: 1789-1796, 2008.

18. Kaseb AO, Chinnakannu K, Chen D, Sivanandam A, Tejwani S, Menon M, Dou QP and Reddy GP: Androgen receptor and E2F-1 targeted thymoquinone therapy for hormone-refractory prostate cancer. Cancer Res 67: 7782-7788, 2007.
19. Dirican A, Erten C, Atmaca H, Bozkurt E, Kucukzeybek Y, Varol U, Oktay Tarhan M, Karaca B and Uslu R: Enhanced cytotoxicity and apoptosis by thymoquinone in combination with zoledronic acid in hormone-and drug-resistant prostate cancer cell lines. J BUON 19: 1055-1061, 2014

20. Iskender B, Izgi K, Hizar E, Jauch J, Arslanhan A, Yuksek EH and Canatan H: Inhibition of epithelial-mesenchymal transition in bladder cancer cells via modulation of mTOR signalling. Tumour Biol 37: 8281-8291, 2016.

21. Zhou Q, Zheng X, Chen L, Xu B, Yang X, Jiang J and Wu C: Smad2/3/4 pathway contributes to TGF- $\beta$-induced miRNA-181b expression to promote gastric cancer metastasis by targeting Timp3. Cell Physiol Biochem 39: 453-466, 2016.

22. Zimmerman KA, Xing D, Pallero MA, Lu A, Ikawa M, Black L, Hoyt KL, Kabarowski JH, Michalak M and Murphy-Ullrich JE: Calreticulin regulates neointima formation and collagen deposition following carotid artery ligation. J Vasc Res 52: 306-320, 2015.

23. Ren X, Bo Y, Fan J, Chen M, Xu D, Dong Y, He H, Ren X, Qu R, Jin Y, et al: Dalbergioidin ameliorates doxorubicin-induced renal fibrosis by suppressing the TGF- $\beta$ signal pathway. Mediators Inflamm 2016: 5147571, 2016.

24. Tennant BR, Chen J, Shih AZ, Luciani DS and Hoffman BG: Myt3 mediates laminin-V/integrin- $\beta 1$-induced Islet-cell migration via Tgfbi. Mol Endocrinol 29: 1254-1268, 2015.

25. Zhao J, Cheng Q, Ye P, Yang G, Liu S, Ao Q, Liu Y and Hu Y: Atorvastatin improves pathological changes in the aged kidney by upregulating peroxisome proliferator-activated receptor expression and reducing matrix metalloproteinase- 9 and transforming growth factor- $\beta 1$ levels. Exp Gerontol 74: 37-42, 2016.

26. Zhang L, Cheng X, Gao Y, Zhang C, Bao J, Guan H, Yu H, $\mathrm{Lu} \mathrm{R}, \mathrm{Xu} \mathrm{Q}$ and Sun Y: Curcumin inhibits metastasis in human papillary thyroid carcinoma BCPAP cells via down-regulation of the TGF- $\beta / \mathrm{Smad} 2 / 3$ signaling pathway. Exp Cell Res 341: $157-165,2016$.

27. Ouanouki A, Lamy S and Annabi B: Anthocyanidins inhibit epithelial-mesenchymal transition through a TGF $\beta / \mathrm{Smad} 2$ signaling pathway in glioblastoma cells. Mol Carcinog 56: 1088-1099, 2017. 